\title{
Auch polnische Schüler denken, forschen und gewinnen moralisch- demokratische Kompetenzen. Projekt-Bericht
}

\section{Anna Leśniewska \& Mateusz Bonecki (Poznań)}

Im jungen demokratischen Polen finden in der letzten Zeit heftige Diskussionen über die demokratischen Bildung und deren Methoden: gleichzeitig wird die Kondition polnischen Schulsystems ins Visier genommen. Vor allem wirft man der polnischen Schule, about it dass sie ihre Absolventen ohne grundlegende kognitive und praktische Kompetenzen frei lässt: besonders knapp sind da selbständiges Denken und Problemlösenfähigkeit. Ehemalige Schüler geben sich ratlos und zum Leben in der Demokratie kaum fähig; dies betrifft ach ihr Berufsleben und Teilnahme an den demokratischen Institutionen. Die erste Diagnose ist bitter: die polnische Schule geht dogmatisch um nicht nur mit dem Religionsunterricht (der nach wie vor keine Alternative im Ethikunterricht findet), buy information pills sondern sogar mit der Wissenschaft, wo der Fortschritt enorm schnell ist. Die Pädagogen sind zutiefst dazu überzeugst, dass Lernen und Bildung kumulative Prozesse sind und auf der kritiklosen Aufnahme und Aneignung enzyklopädischer Informationsmenge beruhen. Der Religionsunterricht besteht aus Indoktrination, sprich: eindimensionaler Werteerziehung als gäbe es keine Normen ausser die katholischen. Die Abwesenheit der Ethik in den Schulprogrammen wird aber immer öfter als der Entwicklung der Demokratie schadend betont. Es gibt ca. 1000 Ethiklehrer in Polen (die jedoch oft kirchlicher Prägung sind); an den etlichen Schulen, wo Ethik überhaupt unterrichtet wird, befindet sie sich in den Händen katholischer Geistlicher.

Im Rahmen unseres Pilot-Projekts Civic Education in the Middle Europe, das seit zwei Jahren am Philosophischen Institut der Adam-Mickiewicz-Universität in Poznań durchgeführt wird, hat sich eine gute Zusammenarbeit zwischen dem Lehrstuhl für Ethik und dem 6. Posener Lyzeum ?Paderek? ergeben. Wir versuchen der Schüler sozio-moralische Schlüsselfähigkeiten wie moralische Urteilskompetenz und Diskurskompetenz zu fördern. Die Förderungsmethoden verdanken wir der Idee von ?Forschungsgemeinschaft? (?Community of Inquiry?, ?Community of Investigators?, ?Philosophieren mit Kindern?) einerseits, und der ?Konstanzer Methode der Dilemma- Diskussion? Georg Linds andererseits. Beide Methoden haben es zum Ziel, die moralische Urteilsfähigkeit bei Kindern und Heranwachsenden zu stärken, und zwar dadurch, dass man durch die affektiven Prozesse (etwa moralische Gefühle, dem Wundern u.ä.) das Verstehen und die Reflexion aktiviert, und dies zwar in einem freien didaktischen Diskursraum, der den Teilnehmern von uns geboten wird.

?Philosophieren mit Kindern? und die ?Konstanzer Methode? teilen dieselbe Vorstellung, dass demokratische Bildung nicht zur Vermittlung der Fachkenntnisse und Fertigkeiten reduziert werden darf, sondern sie muss auch die Entwicklung moralischdemokratischer Fähigkeiten erstreben. Nach Georg Lind (2009), dem Urheber der ?Konstanzer Methode der DilemmaDiskussion? Demokratie sei mehr als eine Regierungsform, denn sie sei ?im Kern eine moralische Institution?. Der Begriff Demokratie umfasst soziale und ethische Praktiken. Auch die Anhänger des inzwischen paradigmatisch gewordenen ?Philosophieren mit Kindern? sind davon überzeugt, dass die Umwandlung des traditionellen Klassenzimmers zur spannenden Community of Inquiry stark zur Entwicklung moralisch-demokratischen beitragen kann. Interessierte, forschende Kinder beantworten philosophische Fragen, wo man mit mehreren Antworten rechnen muss und dadurch u.a. gegenseitigen Respekt, Pluralismus und Kooperation lernt. Offenes gemeinsames Nachdenken wird auch nicht durch autoritäres Verhalten ?traditionellen? Lehrers gestört. Demokratischer Dialog und demokratisches Erlebnis sind spannend und dazu machen sie noch Spass, was im Schulleben nicht immer der Fall ist.

Im 6. Lyzeum haben wir im September 2009 mit einem ?Philosophie-Stunde? Zyklus angefangen (bis Juni 2010). Zu Ende dieses Programms mit vielen Diskussionselementen und der Einführung in die lebensnahen Bereiche der Philosophie, haben wir mit drei Klassen (17- und 18-jährige Schüler) mehrere Dilemma-Diskussionen durchgeführt. Da, wo die Schüler einstimmig PRO oder CONTRA abgestimmt haben, wurde gleich eine andere, philosophische oder ethische Diskussionsrunde durchgeführt (es war auch den Lehrern besonders zu recht, da sie bei den Abituren beschäftigt waren).

Im Hintergrund beider erwähnten Methoden der Kompetenzförderung steht gewisse Überlieferung: sie ist in den beiden Fällen durchaus Sokratisch und Aristotelisch. Im Dialog und aktiven Erwägen sowie Erläutern moralischer Kontroverse kommen die Teilnehmer immer ein Stück weiter. Sie tauschen ihre verschiedenen Meinungen aus, argumentieren und stellen ihre Meinungen in Frage. So werden nicht nur die moralische Sprache und Reflexion entwickelt, sondern auch kooperatives und zugleich kritisches (selbstkritisches) Denken. Zur Aufklärungszeit wurde die Sokratische Tradition wieder stark: man hat erneut entdeckt, dass der moralische Lebensstil einer unabhängigen Reflexion und Selbstreflexion benötigt. In der Epoche der praktischen Vernunft und des transzendentalen Ichs, welches dem empirischen (entscheidenden, handelnden und erfahrenden) überlegen ist, indem es die richtige Instanz des (ethischen und kritischen) Selbstbewusstseins ist. Dem Menschen kommt nicht nur die gleiche Vernünftigkeit und Autonomie, die jedoch entwickelt werden müssen durch Bildung, sondern auch der angeborene ?moralische Sinn? (Hume), der kultiviert werden muss. Auf diese Erkenntnis war auch Sokrates mit seiner Idee von anamnesis eines angeborenen Ur-Wissens gekommen. Diese Ideale prägen die Konstanzer Methode der DilemmaDiskussion und ?Inquiry?. 
In der pragmatischen Tradition (insb. von Peirce und Dewey) besteht die Aufgabe der Forschung und Untersuchung (Inquiry) darin, dass die konkreten alltäglichen Probleme angesprochen werden, und dies zur aktiven Verbesserung des gesellschaftlichen Lebens der Lebensqualität ihrer Mitglieder (Glaser, 2007). Die Zielsetzung sowie das Programm unserer ?Philosophie-Stunde Zyklus? sind den beiden Traditionen: der Sokratischen und der pragmatischen, getreu.

Schon die Bezeichnung ?Philosophie-Stunde? anstelle ?einer Stunde mit der Philosophie? verweist daraufhin, dass unsere Hauptabsicht nicht gerade die philosophische Theorie ist, sondern ein praktizierbares Philosophieren, das nach der Auffassung Ekkehard Martens' sich durch die Einheit von Haltung (Neugier und Offenheit), Methode (kritische, begrifflich-argumentative Prüfung) und Inhalt (philosophische Themen) kennzeichnet. Martens betont, es gebe ?keine typisch philosophischen Fragen, sondern nur Fragen, die durch die charakterisierte Haltung und Methode zu philosophischen Fragen werden können: im philosophischen Dialog geht es zunächst um ein ganz konkrete Alltagsfrage, die sich erst im gemeinsamen, (durch Sokrates z. B. angeregten) Prozess des Weiterdenkens zu einer grundsätzlichen Frage verändert? (Martens, 1999, S. 13).

Während einer Philosophie-Stunde, die meistens eine diskursive Form annahm, spielte der Gesprächsauslöser (Impuls) eine ganz wichtige Rolle: es waren sorgfältig auserwählte philosophische Aussagen mit dem immanenten Fragenpotential wie z. B. die Aphorismen von Heraklit, die Platonischen Dialoge, literarische Texte und die Themen und Motive aus der gängigen Popkultur. Es ging vor allem darum, dieses Arbeitsmaterial philosophisch zu behandeln, nämlich analytisch, kritisch, ?begründungsbedürftig?. Allen unterrichtenden Personen war es auch wichtig, an den von der Gruppe als problematisch erlebten Fragen im Medium argumentativer Gespräche, im gemeinsamen Dialog, besonders gründlich zu arbeiten. Als entscheidend haben sich hier die Aufgaben des Lehrers erwiesen: dialogische Gemeinschaft benötigt eines dezenten Moderators, der mit seiner Autorität nicht demütigend wirkt, sondern verzichtet auf diejenige um die jungen Diskutanten zum freien Denken und Deliberieren zu fördern. Die Teilnehmer unserer ?Stunden? sowie ihr Lehrpersonal, welches unseren Unterricht oft beobachten konnte, hat es besonders hoch geschätzt, dass die Moderatoren keine Autoritätsansprüche erhoben haben. Das ermutigte die Schüler und sorgte um die vertrauensvolle Atmosphäre im Klassenzimmern.

Auch der Feedback nach den Dilemmadiskussionen war positiv: den Schülern hat das affirmative Verhalten der KMDD-Lehrer gefallen die ?keinen beurteilt haben?. ?Es gab eine untypische Unterrichtspraxis, in der jeder seine Meinung offen ausdrücken konnte?. Die Teilnehmer haben auch die ?ganz wichtige Rolle der Ping-Pong Regel? betont, die erlaubte ?vielen Schüler zum Wort zu kommen?. Allerdings haben die Beobachter unserer Dilemmadiskussionen darauf aufmerksam gemacht, dass die Diskutanten allesamt problemlos an beiden KMDD-Regeln (also der des gegenseitigen Respekts) hielten.

Die Stimmen der Beobachter und die der KMDD-Teilnehmer überschnitten sich auch in dem Punkt, dass der

Meinungsaustausch enorm intensiv war und ?die Vielfalt und Vielheit der Standpunkte und insb. der Argumente für jede Position? zu sehen war (so die Teilnehmer). Nach einer der KMDD-Diskussionen hat ein Junge gesagt, dass er ?die Hauptidee überhaupt gut gefunden habe?, und zwar ?dass wir über das alltägliche Problem diskutiert haben?, obwohl (so eine weitere Meldung in derselben Gruppe): ?uns das Fachwissen fehlte, wie man ein solches Problem recht lösen sollte.? Dies aber verweist wieder daraufhin, dass das Schulsystem darauf basiert, den Schülern die falsche Idee beizubringen, dass es in der Ethik bzw. Moraltheorie fertige Lösungen gebe und dass das Individuum selbst vom Denken und Suchen nach einer situationsorientierten Lösung befreit ist. Dies hat uns vergewissert, dass wir unseres Programm in den kommenden Jahren fortsetzen und womöglich erweitern müssen.

\section{Literatur}

Freese, H.-L. (1989), Kindern sind Philosophen, Berlin: Weinheim.

Glaser, J. (2007). Educating for Citizenship \& Social Justice, in: D. G. Camhy (Ed.), Philosophical Foundation of Innovative Learning. Proceedings of the International Conference on Philosophy for Children in Graz, Austria, Sankt Augustin: Academia Verlag, pp. 16?24.

Habermas, J. (1983). Moralbewusstsein und Kommunikatives Handeln, Frankfurt am Main: Suhrkamp.

Lind, G. (2009). Moral is lehrbar. Handbuch zur Theorie und Praxis moralischer und demokratischer Bildung. Oldenbourg: München.

Lipman, M. (1993). Thinking Children and Education, Dubuque: Kendall/Hunt.

Lipman, M. (1991). Thinking in Education, Cambridge: Cambridge University Press.

Lipman, M., Sharp, A. M., Oscanyan, F. S. (1980). Philosophy in the Classroom, Philadelphia: Temple University Press.

Martens, E. (1999). Philosophieren mit Kindern. Eine Einführung in die Philosophie, Stuttgart: Philipp Reclam.

Matthews, G. B. (1989). Philosophische Gespräche mit Kindern, Berlin: Freese Verlag. 
Sharp, A. M. (2007). The Classroom Community of Inquiry and the Education of Emotions, in: D. G. Camhy (Ed.), Philosophical Foundation of Innovative Learning. Proceedings of the International Conference on Philosophy for Children in Graz, Austria, Sankt Augustin: Academia Verlag, pp. 84?94. 